\title{
Correlation scales of digital elevation models in developed coastal environments
}

\author{
Christopher Small ${ }^{\mathrm{a}, *}$, Robert Sohn ${ }^{\mathrm{b}}$ \\ a Lamont-Doherty Earth Observatory, Columbia University, Palisades, NY 10964, USA \\ b Woods Hole Oceanographic Institution, Woods Hole, MA 02543, USA
}

\section{A R T I C L E I N F O}

\section{Article history:}

Received 17 September 2013

Received in revised form 19 November 2014

Accepted 21 November 2014

Available online 29 December 2014

\section{Keywords:}

Digital elevation model

DEM

LiDAR

SRTM

GDEM2

Urban

Wetland

Coastal

Topography

\begin{abstract}
A B S T R A C T
Accuracy of digital elevation models (DEMs) often depends on how features of different spatial scales are represented. Scale dependence is particularly important in low gradient coastal environments where small vertical errors can affect large areas and where representation of fine scale topographic features can influence how DEMs are used for modeling inundation. It is commonly observed that different types of DEMs represent larger, coarse-scale topographic features similarly but differ in how they represent smaller, finer-scale features. The spatial-scale dependence of DEM accuracy can be quantified in terms of the correlation scale $\left(\lambda_{c}\right)$; the spatial wavelength above which models agree with spectral coherency $>0.5$ and below which they differ. We compare cross spectral analyses of the GDEM2 and SRTM global DEMs with 14,572 LiDAR-derived elevations along transects in diverse coastal environments of New York City. Both global DEMs have positive bias relative to LiDAR ground elevations, but bias $(\mu)$ and uncertainty $(\sigma)$ of GDEM2 $(\mu: 8.1 \mathrm{~m} ; \sigma: 7.6 \mathrm{~m})$ are significantly greater than those of SRTM ( $\mu: 1.9 \mathrm{~m}$; $\sigma: 3.6 \mathrm{~m}$ ). Cross-spectral coherency between GDEM2 and the LiDAR DEM begins to roll-off at scales of $\lambda<\sim 3 \mathrm{~km}$, while coherency between SRTM and the LiDAR DEM begins to roll-off at scales of $\lambda<\sim 1 \mathrm{~km}$. The correlation scale below which coherency with LiDAR attains a signal to noise ratio of 1 is $\sim 1 \mathrm{~km}$ for GDEM2 and $\sim 0.5 \mathrm{~km}$ for SRTM; closely matching the divergence scales where the surface roughness of the land cover exceeds the roughness of the underlying terrain.
\end{abstract}

@ 2014 Published by Elsevier Inc.

\section{Introduction}

Hazard assessments and inundation modeling of coastal areas rely heavily on both the accuracy and resolution of digital elevation models (DEMs). In many coastal areas, global DEMs offer the most complete representation of coastal elevations and morphology available. Two distinct classes of global DEM are currently in widespread use: passive source stereographic models derived from optical imagery like the ASTER GDEM2 (Abrams et al., 2010) and active source ranging models derived from synthetic aperture radar like the SRTM (Farr et al., 2007). The accuracy of each model depends on multiple factors related to the sensing modality, the procedure used to estimate elevations, and the characteristics of the land surface (Farr et al., 2007; Lang \& Welch, 1999). The recent release of full-resolution $30 \mathrm{~m}$ SRTM data for areas outside the US (previously degraded to $90 \mathrm{~m}$ ) prompts the question of how the accuracy and effective spatial resolution of SRTM and GDEM2 compare, particularly in developed coastal environments where they may be used for inundation modeling and hazard assessments.

\footnotetext{
* Corresponding author at: 304b Oceanography Lamont Doherty Earth Observatory Columbia University Palisades, NY 10964, USA.

E-mail address: csmall@columbia.edu (C. Small).
}

The accuracy and resolution of DEMs in coastal environments, where there are relatively small differences in elevation over large areas, are of special interest. At low elevations and gradients the signal magnitude approaches the noise level of the measurements, which can lead to large errors in inundation extent forecasts. This issue is particularly important for developed coastal environments where the spatial extent of inundation can have disproportionate consequences in terms of loss of life and property. There have been several comparative analyses of global DEM vertical accuracy (e.g., Gesch et al., 2012; Meyer et al., 2012; Tachikawa et al., 2011; Tadono et al., 2012; Smith \& Sandwell, 2003). Some analyses have included coastal areas (e.g., (Gorokhovich \& Voustianiouk, 2006; Hvidegaard et al., 2012), and some have incorporated land cover/use information (e.g., Gesch et al., 2012; Hofton et al., 2006; Carabajal \& Harding, 2006), but we are not aware of any that specifically consider the accuracy and spatial resolution of global DEMs in developed coastal environments. As explained below, the scale and diversity of land cover in developed coastal areas is fundamentally different from most of the environments where previous studies have focused.

The objective of this analysis is to assess the accuracy and scale dependence of the GDEM2 and SRTM global DEMs in developed coastal environments. We address the issue by quantifying the scale dependence of the agreement between these global DEMs and high- 
accuracy, high-resolution, LiDAR-derived elevations for a diverse variety of coastal environments within New York City (NYC). We quantify the scale dependence by using cross-spectral analysis to estimate the correlation scale (the length scale below which two signals are uncorrelated) of each global DEM with a co-registered DEM and digital surface model (DSM) derived from LiDAR. The LiDAR DEM (LDEM) and DSM (LDSM) have been thoroughly validated throughout the study area and thus provide high-quality benchmarks for the analysis. We focus on quantifying the lateral length-scale at which the agreement between two models becomes random. This is complementary to, but distinct from, previous studies that used point-to-point comparisons (e.g., GPS or fiducial) to measure the absolute accuracy of the global DEMs. To our knowledge, the only scale-dependent analyses of global DEMs are those of (Smith \& Sandwell, 2003; and Rodriguez et al., 2006) but neither focus on developed or coastal environments.

\section{Data}

The geological and geomorphic diversity of NYC includes a wide range of developed and natural coastal environments and land
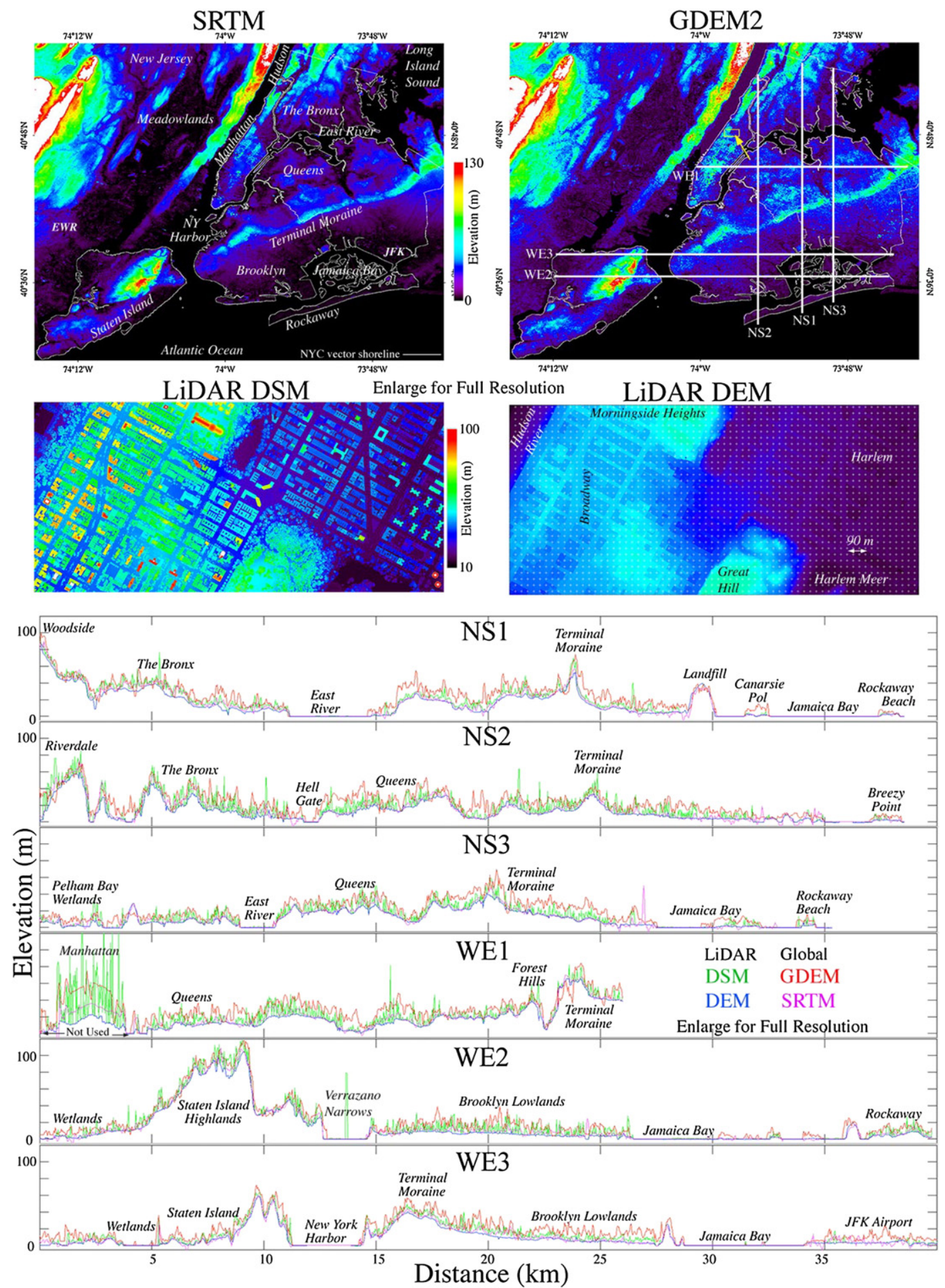

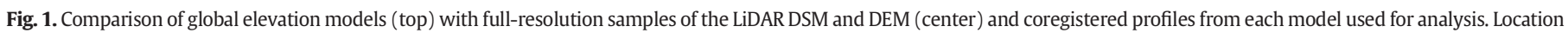
of LiDAR sample shown by arrow and box on GDEM2 map. 
cover/use types. Four of NYC's five boroughs are islands. Included in NYC's $837 \mathrm{~km}$ of coastline are beaches, barrier islands, tidal wetlands, estuarine marshes, rivers, channels and a variety of reinforced embankments on the shores of New York Harbor, Long Island Sound, the Atlantic Ocean and the Hudson, Harlem and East Rivers (Fig. 1).

The LiDAR DEM and DSM were produced from a 17-day airborne campaign conducted by Sanborn Inc. in April 2010. The ALS-50 near infrared LiDAR instrument was flown at an altitude of $1100 \mathrm{~m}$ with a scan angle of $28^{\circ}$. The $937 \mathrm{~km}^{2}$ collection of $15 \times 10^{9}$ elevation measurements results in a point density of 8 to 12 points $/ \mathrm{m}^{2}$. Comparison of LiDAR elevations with 1722 survey elevations throughout NYC yields a root mean square error (RMSE) of $0.075 \mathrm{~m}$ in elevation. Comparison with 200 building corner points yields an RMSE of $0.33 \mathrm{~m}$ horizontal error (Ahern \& Ahn, 2011). Extraction of first and last returns from the full waveform LiDAR allowed for better discrimination of true ground elevations under tree canopies and semi-penetrable land cover types. The LDSM measures tops of buildings, trees and infrastructure, as well as ground elevations where sufficient skyview exists. Known ground elevations were used to extract the DEM from the LDSM and last returns. The LDEM ground elevations under buildings are estimates based on surrounding true ground elevations. Some residual building footprints remain in the LDEM (Fig. 1), but their amplitude is generally $<1 \mathrm{~m}$.

The GDEM2 and SRTM (v2.1) DEMs were obtained from the USGS in the form of 1 arc second $\left(1^{\prime \prime}=\sim 28 \mathrm{~m}\right.$ at $\left.40^{\circ} \mathrm{N}\right)$ resolution geographic grids in the WGS84 horizontal and EGM96 vertical reference systems. The LDEM and LDSM were spatially averaged with a 1" Gaussian kernel and sampled at points coincident with the global DEMs along 6 transects (Fig. 1). Transects were chosen to maximize length, relief and diversity of coastal environments. For each transect, two adjacent rows (or columns) of the global DEMs were compared to check consistency of spectral estimates for similar (but not identical) realizations of the terrain. Transect WE1 extends westward from the East River through midtown Manhattan where neither global DEM was able to register accurate elevations because of the considerable height variability resulting from tall buildings, as can be seen in the LiDAR DSM. The NUM files that accompany SRTM v3 indicate that infill elevations were used to fill coverage gaps in midtown Manhattan and one pol on Jamaica Bay but not any other part of the transects selected for analysis. We exclude midtown from our spectral analyses but include it in Fig. 1 to illustrate the infill elevations used to fill the gaps in DEM coverage. Within the NYC study area, the SRTM grid used between one and four (mode $=3$ ) acquisitions and GDEM2 used between one and twelve (mode $=7$ ) acquisitions.

\section{Analysis and results}

We use scatterplots and moments of difference distributions to quantify the point-to-point correspondence between the global DEMs and the 14,572 LiDAR elevations. The results show closer agreement between LDEM and SRTM than between LDEM and GDEM2 (Fig. 2). In the LDEM and SRTM comparison, 25\% of the SRTM elevations underestimate the LiDAR ground elevations while almost all of the GDEM2 elevations overestimate the LiDAR ground elevations. An important difference between global DEMs is observed at the lowest elevations, where GDEM2 always overestimates LDEM but SRTM often
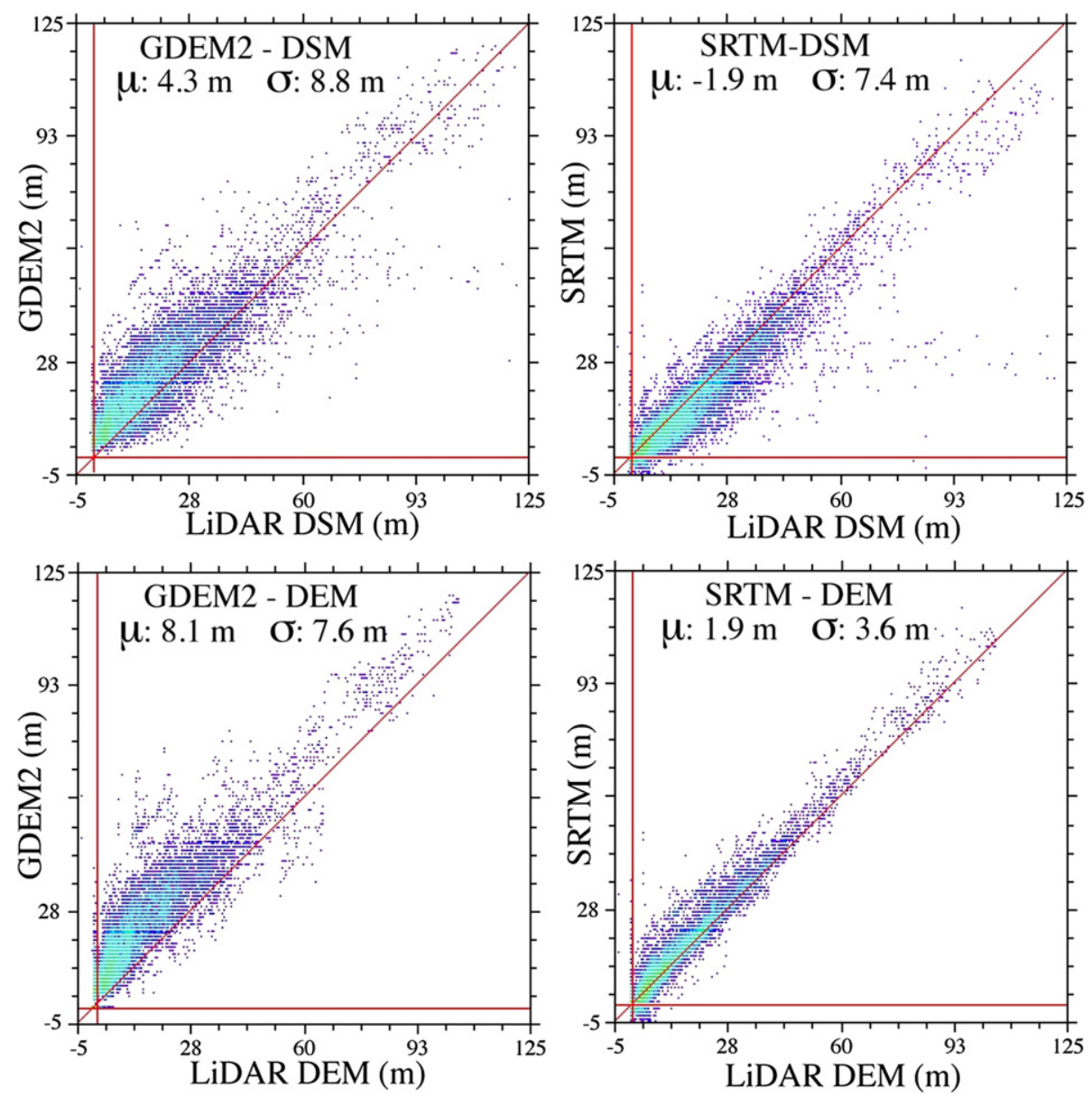

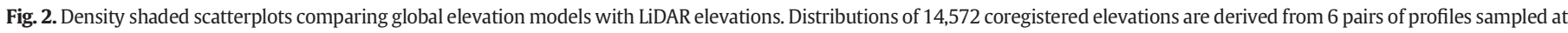

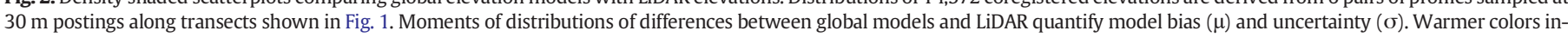
dicate greater number of points. 
underestimates LDEM (Fig. 2). This difference is also illustrated by the large, low elevation wetlands (e.g., Jamaica Bay and New Jersey Meadowlands) in Fig. 1. The 1st and 2nd moments of the difference distributions give the bias $(\mu)$ and uncertainty $(\sigma)$ of each global DEM relative to the corresponding LiDAR model. Both global DEMs have overall positive bias relative to the LiDAR DEM, but GDEM2 bias and uncertainty are much greater than those of SRTM (Fig. 2).

We conduct cross-spectral analyses of the four elevation models along all 6 transects to assess the scale dependence of correlation. Power spectral density, cross-spectral phase and spectral coherency are estimated using the multitaper method (Thomson, 1982) with adaptive weighting (Percival \& Walden, 1993) and a time-bandwidth product of eight. Multitaper estimation reduces the bias resulting from spectral leakage while minimizing the information loss inherent in the use of conventional tapers and avoiding the need for prewhitening (Thomson, 1982). Adaptive weighting minimizes the mean square error of the spectral estimates by determining the weights for each taper using an iterative procedure that accounts for the (nonwhite) spectral content of the data (Percival \& Walden, 1993).

The power spectral density estimates show the relative amount of variance over a range of spatial scales (wavelengths). Power spectra of elevation data are often used to quantify scale dependent variance as a proxy for topographic roughness (e.g., Fox \& Hayes, 1985). The spectral shape and roll-off of each transect are functions of the elevation profile roughness (Fig. 3, top panels). For example, LDSM consistently has more power than LDEM at short wavelengths because the land cover upper surface (e.g., buildings and trees) is rougher than the underlying topography, especially in developed areas with multi-story buildings. GDEM2 consistently has greater power than SRTM at short wavelengths, perhaps because of noise in GDEM2 resulting from spectral heterogeneity of land cover imaged by ASTER. Spectral power levels for all four elevation models are similar for length scales $>\sim 1 \mathrm{~km}$ but begin to diverge at length scales of $0.5-1.0 \mathrm{~km}$, where the relief associated with land cover (e.g., buildings and trees) has greater variance than the smoother underlying topography. We refer to this wavelength where the DSM and DEM separate (shown by arrows in Fig. 3 ) as the surface-elevation divergence scale $\left(\lambda_{\mathrm{D}}\right)$.

The cross-spectral analysis (Fig. 3, bottom panels) indicates that both global DEMs become incoherent at spatial scales $<\sim 0.5 \mathrm{~km}$. Coherency, which estimates correlation as a function of spatial wavelength, between GDEM2 and both LiDAR models begins to roll off for wavelengths $<\sim 3 \mathrm{~km}$, while the coherency between SRTM and LDEM begins to roll off for wavelengths $<\sim 1 \mathrm{~km}$. We define the correlation scale $\left(\lambda_{C}\right)$ between two models as the length scale at which the coherency drops below 0.5 , a threshold value representing a signal-to-noise ratio of one (Bendat \& Piersol, 2010). We find that the correlation scale for GDEM2 relative to both LiDAR models is between 1.4 and $0.7 \mathrm{~km}$, while the correlation scale for SRTM relative to LDEM is between 0.7 and $0.4 \mathrm{~km}$. These correlation scales $\left(\lambda_{C}\right)$ are similar to the surface-elevation divergence scales $\left(\lambda_{D}\right)$ observed in the power spectra (Fig. 3). This indicates
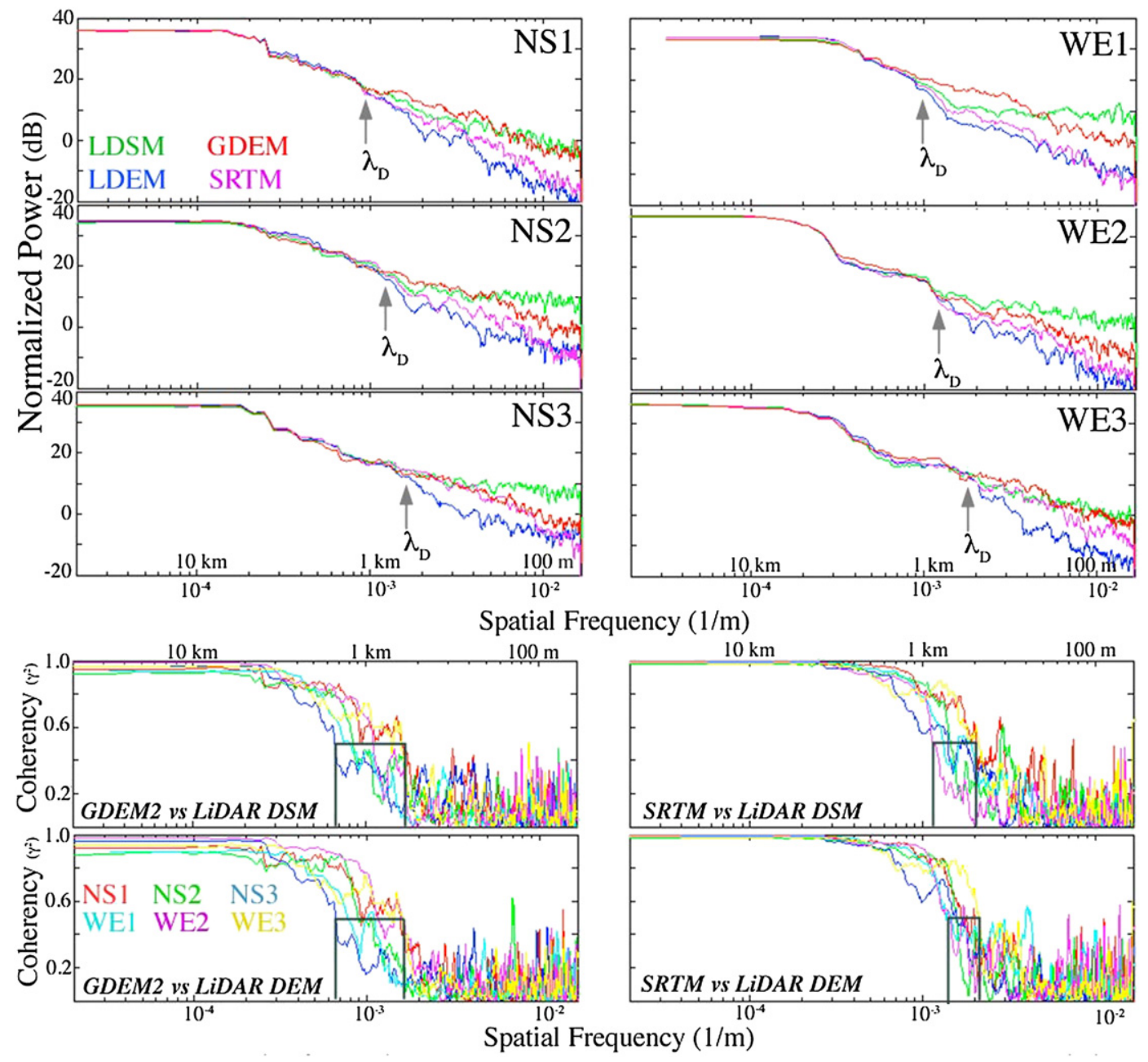

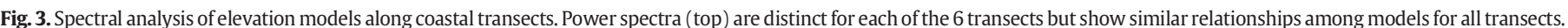

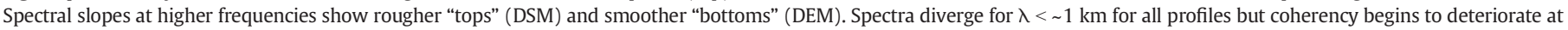

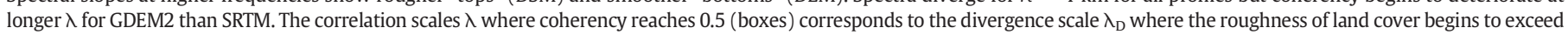
the roughness of the underlying terrain (arrows). 
that the correlation scales where agreement among DEMs disappears occurs at divergence scales where the surface roughness of the land cover exceeds the roughness of the underlying terrain.

The cross-spectral analysis suggests that structure at scales $<\sim 1 \mathrm{~km}$ is as likely to be noise as true elevation in both global DEMs. To illustrate this, we filter all four DEMs for profile NS2 to remove incoherent structure at wavelengths $<1 \mathrm{~km}$ (Fig. 4). The filtered profiles are in much closer agreement than the raw profiles, especially for the SRTM and LDEM, and the positive bias of GDEM2 is readily apparent.

\section{Implications}

Our results quantify the correlation scales of the GDEM2 and SRTM global elevation models for both developed and natural coastal environments in NYC. In general, we find that the global DEMs accurately resolve features with length scales $>\sim 1 \mathrm{~km}$, but at shorter length-scale noise overwhelms the elevation signal. The correlation scale for SRTM extends to shorter length scales $(\sim 500 \mathrm{~m})$ compared to GDEM2 $(\sim 1 \mathrm{~km})$, and GDEM2 exhibits a systematic, $\sim 8 \mathrm{~m}$ positive bias throughout the study area (Figs. 1 and 2). This may be due, in part, to vertical reference error in GDEM2.

The power spectra and correlation scales vary somewhat among the profiles, with most differences occurring at spatial scales finer than $\sim 1 \mathrm{~km}$ where the land cover and the underlying topography signals begin to diverge. The accuracy of the global DEMs begins to deteriorate at about the same length-scale where heterogeneous land cover associated with developed environments becomes the dominant signal. The consistently higher variance (power) and lower coherencies we observe for GDEM2 compared to SRTM for length scales of $0.5-3 \mathrm{~km}$ suggest that heterogeneous land cover in developed areas introduces more noise into GDEM2 elevation estimates compared to SRTM elevation estimates.

The scale and diversity of urban land cover poses challenges to stereography using decameter resolution imagery. Comparative multi-scale analyses of meter to decameter resolution optical imagery of urban environments reveal considerable intra-urban spectral diversity with characteristic spatial scales of 20 to $50 \mathrm{~m}$ (Small, 2009). The spectral diversity and scale-dependent spectral mixing endemic to urban land cover violates the assumptions of spectral homogeneity and Lambertian scattering that are implicit to stereography (Lang \& Welch, 1999). As a result, the 20-30 m spatial resolution of the ASTER sensor is not well suited to stereography in heterogeneous urban environments with abundant specular reflectors. However, this does not imply that GDEM2 is not well suited to more spectrally homogeneous environments with greater topographic relief. GDEM2 is an important complement to SRTM because it provides coverage at higher latitudes and in areas of very steep terrain and sand dunes where SRTM often contains voids (Farr, 2006).

The correlation scales of the SRTM and LDEM are consistent with, but larger than, previous studies that quantify scale-dependent resolution of SRTM. The continental-scale structure function analyses conducted by Rodriguez et al. (2006) find height error correlation functions dropping rapidly for scales $<\sim 500 \mathrm{~m}$. The cross spectral analysis of LiDAR and SRTM for Mojave Desert terrain conducted by Smith and Sandwell (2003) found average spectral coherence of 0.5 at scales of $\sim 200 \mathrm{~m}$. The effective spatial resolution of the stereographic and synthetic aperture radar algorithms is generally coarser than the $30 \mathrm{~m}$ grid resolutions but finer than the 0.5 to $1 \mathrm{~km}$ correlation scales observed in this study. The larger correlation scales found in this study suggest that global DEMs may have lower effective spatial resolution (or that noise levels are correspondingly higher) in developed coastal environments compared to continental averages and mountainous, high desert, environments. We conjecture that the lower effective spatial resolutions of both global DEMs in developed coastal environments is a result (at least in part) of the heterogeneity of land cover with characteristic spatial scales comparable to the IFOV of the sensors used for the global models.

Overall, both global DEMs are well within their stated accuracy specifications and appear to have closer agreement to measured elevations than in some previous studies cited above. However, in addition to the apparently random errors discussed above, we do observe some systematic errors when comparing full 2D DEMs at 1 " resolution. The largest and most obvious errors in both models occur in areas of high relief at pixel scales (e.g., buildings in Manhattan). Both global DEMs overestimate ground elevation and underestimate building top heights by

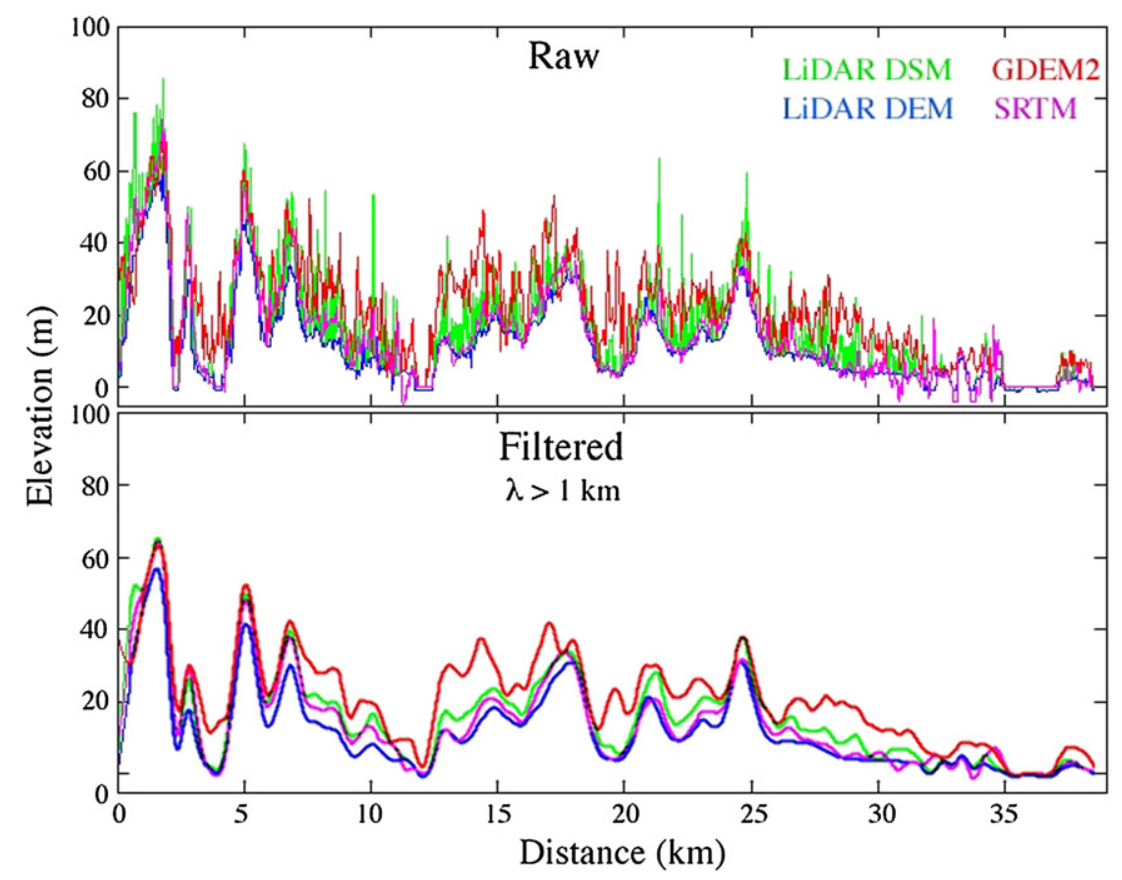

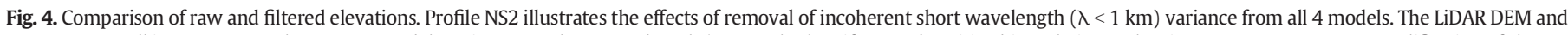

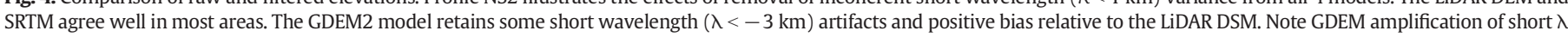
features of DSM. 
$>10 \mathrm{~m}$ in most areas where buildings exceed tree height. Consistent with the findings of (Hofton et al., 2006), SRTM overestimates elevation in areas of dense, closed canopy forest where the phase center lies within the canopy. GDEM2 also overestimates surface elevations in some wetlands.

\section{Acknowledgments}

This research was supported by the Office of Naval Research (grant N000-14-11-1-0653 to C.S.). We thank Tom Farr and Bob Crippen for helpful comments and suggestions. ASTER GDEM is a product of METI and NASA.

\section{References}

Abrams, M., Bailey, B., Tsu, H., \& Hato, M. (2010). The ASTER global DEM. Photogrammetric Engineering and Remote Sensing, 76, 344-348.

Ahern, S.C., \& Ahn, H.J. (2011). Quality assurance and potential applications of a high density LiDAR data set for the City of New York. Proceedings of ASPRS (pp. 9).

Bendat, J.S., \& Piersol, A.G. (2010). Random data: Analysis and measurement procedures (4th Edition ). Wiley.

Carabajal, C.C., \& Harding, D.J. (2006). SRTM C-band and ICESat laser altimetry elevation comparisons as a function of tree cover and relief. Photogrammetric Engineering and Remote Sensing, 72, 287-298.

Farr, T.G. (2006). Voids in SRTM data caused by sand dunes. EUSAR 2006-6th European Conference on Synthetic Aperture Radar. Dresden, Germany.

Farr, T.G., Rosen, P., Caro, E., Crippen, R., Duren, R., Hensley, S., et al. (2007). The shuttle radar topography mission. Reviews of Geophysics, 45, 1-33.

Fox, C.G., \& Hayes, D.E. (1985). Quantitative methods for analyzing the roughness of the seafloor. Reviews of Geophysics, 23, 1-48.
Gesch, D., Oimoen, M., Zhang, Z., Meyer, D., \& Danielson, J. (2012). Validation of the aster global digital elevation model version 2 over the conterminous United States. 2012 XXII ISPRS Congress (pp. 281-286). Melbourne, Australia: ISPR.

Gorokhovich, Y., \& Voustianiouk, A. (2006). Accuracy assessment of the processed SRTMbased elevation data by CGIAR using field data from USA and Thailand and its relation to the terrain characteristics. Remote Sensing of Environment, 104, 409-415.

Hofton, M., Dubayah, R., Blair, J.B., \& Rabine, D. (2006). Validation of SRTM elevations over vegetated and non-vegetated terrain using medium footprint lidar. Photogrammetric Engineering \& Remote Sensing, 72, 279-285.

Hvidegaard, S.M., Sorensen, L.S., \& Forsberg, R. (2012). ASTER GDEM validation using LiDAR data over coastal regions of Greenland. Remote Sensing Letters, 3.

Lang, H.R., \& Welch, R. (1999). ATBD-AST-08 algorithm theoretical basis document for aster digital elevation models (standard product AST14). NASA.

Meyer, D.J., Tachikawa, T., Abrams, M., Crippen, R., Krieger, T., Gesch, D., et al. (2012). Summary of the validation of the second version of the aster GDEM. 2012 XXII ISPRS Congress (pp. 291-293). Melbourne, Australia: ISPRS

Percival, D.B., \& Walden, A.T. (1993). Spectral analysis for physical applications. Cambridge, England: Cambridge University Press.

Rodriguez, E., Morris, C.S., \& Belz, J.E. (2006). A global assessment of the SRTM performance. Photogrammetric Engineering \& Remote Sensing, 72, 249-260.

Small, C. (2009). The color of cities: An overview of urban spectral diversity. In M. Herold, \& P. Gamba (Eds.), Global mapping of human settlements (pp. 59-106). Taylor and Francis.

Smith, B., \& Sandwell, D. (2003). Accuracy and resolution of shuttle radar topography mission data. Geophysical Research Letters, 30 (20-21-24).

Tachikawa, T., Hato, M., Kaku, M., \& Iwasaki, A. (2011). Characteristics of ASTER GDEM version 2. International Geoscience and Remote Sensing Symposium (IGARSS) (pp. 3657-3660). IEEE.

Tadono, T. Takaku, J \& Shimada, M. (2012). Validation study on alos prism DSM mosaic and aster GDEM 2. 2012 XXII ISPRS Congress (pp. 193-198). Melbourne, Australia: ISPRS.

Thomson, D. (1982). Spectrum estimation and harmonic analysis. Proceedings of the IEEE, 70, 1055-1096. 